\title{
Expanding approaches to detect clonal hematopoiesis
}

M.A. Wasay Khan and Alexander G. Bick

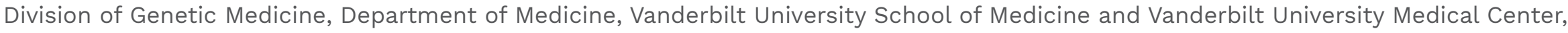
Nashville, TN, USA

E-mail: alexander.bick@vumc.org

https://doi.org/10.3324/haematol.2021.279818

(C)2022 Ferrata Storti Foundation

Haematologica material is published under a CC-BY-NC license @c) (1) \&

Clonal hematopoiesis $(\mathrm{CH})$ occurs when a single hematopoietic stem cell acquires a mutation that gives it a competitive advantage over other stem cells. $\mathrm{CH}$ is often driven by somatic mutations in genes that are recurrently mutated in myeloid malignancies such as DNMT3A, ASXL1, or TET2. $\mathrm{CH}$ is present at an appreciable fraction of the blood in more than $10 \%$ of people older than 70 years and is a precursor to hematologic cancers. The rate of development of neoplasia in patients with $\mathrm{CH}$ is estimated to be approximately $0.5 \%$ to $1 \%$ per year. ${ }^{1}$ The contributing factors to clonal progression other than the selective pressure conferred by the driver mutation ${ }^{2}$ are incompletely understood but proposed mechanisms include inflammation, ${ }^{3}$ short telomeres leading to chromosomal instability, ${ }^{4}$ an aging bone marrow microenvironment that favors expansion of clonal stem cells ${ }^{5}$ and germline genetic predisposition. ${ }^{6}$ Unexpectedly, $\mathrm{CH}$ was also found to increase the risk of diverse forms of cardiovascular disease. ${ }^{7}$ Accumulating evidence supports a mechanism of accelerated atherogenesis because of vascular inflammation driven by clonally derived monocytes and macrophages.

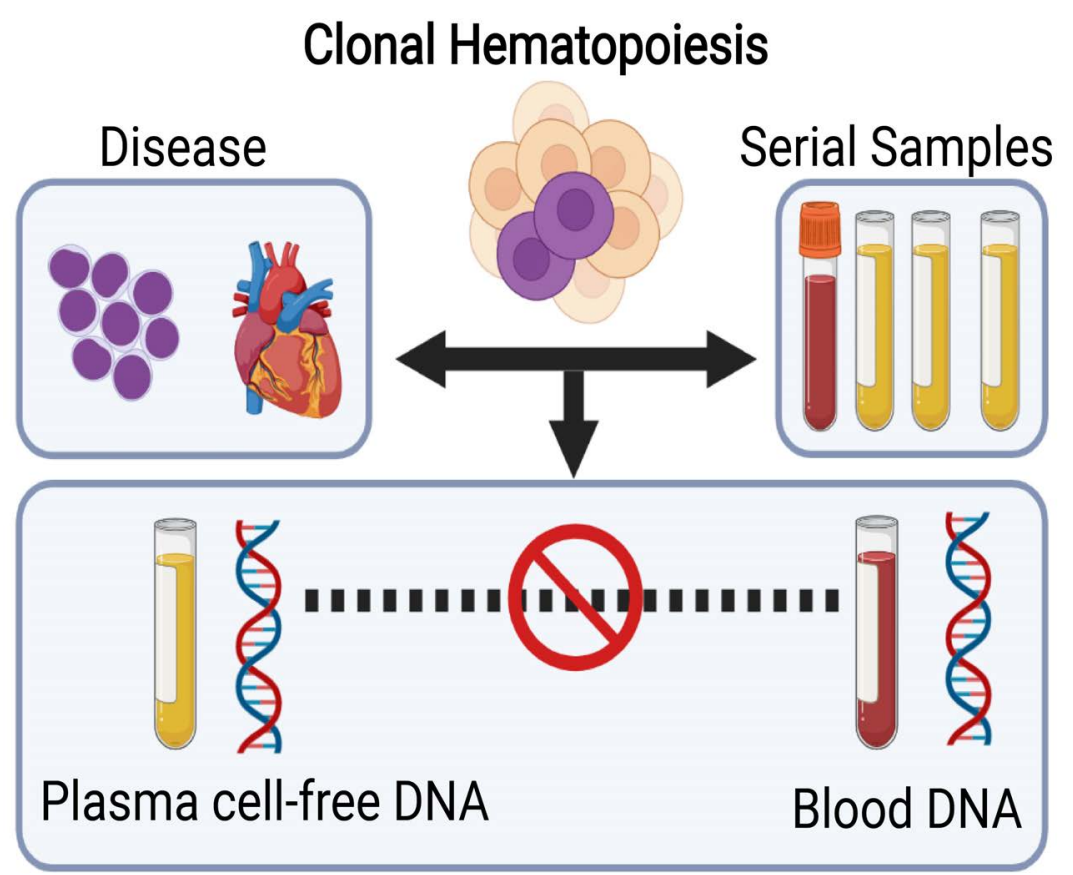

Figure 1. Clonal hematopoiesis is a risk factor for multiple hematologic malignancies and other diseases of aging. Serial plasma samples are often available from large epidemiological cohorts; however, blood is frequently collected at only a single timepoint. Gutierrez-Rodriquez et al. demonstrated that it is possible to detect clonal hematopoiesis from plasma cell-free DNA, but the clonal fraction from cell-free DNA is not always consistent with measurements of clonal hematopoiesis from blood-derived DNA.
Many ongoing studies seek to profile the diverse disease consequences of $\mathrm{CH}$ and how $\mathrm{CH}$ evolution is associated with both malignant and non-malignant disease consequences. Existing collections of samples frequently comprise serial plasma samples to measure changes in biomarkers over time. However, blood for genotyping is usually only collected at a single time point (Figure 1).

Prior studies have observed that plasma cell-free DNA (cfDNA) may contain $\mathrm{CH}$ mutations. For example, $\mathrm{CH}$ is a frequently observed "contaminant" in the context of liquid biopsies when using peripheral blood to detect solid tumor mutations. ${ }^{8}$

In this issue of Haematologica, Gutierrez-Rodriquez et al. ${ }^{9}$ highlight both the potential and shortcomings of utilizing cfDNA to identify individuals with $\mathrm{CH}$. In their study, cfDNA samples from patients with aplastic anemia, myelodysplastic syndrome, and healthy individuals from the Baltimore Longitudinal Study of Aging were screened for somatic variants in $\mathrm{CH}$ driver genes using a chemiluminescent immunoassay commercial targeted sequencing panel. Results were compared to those of DNA derived from paired samples of blood cells from the same individuals. The authors found excellent concordance between cfDNA and paired blood samples among individuals with a hematologic malignancy, particularly with large $\mathrm{CH}$ clones. However, they observed poor concordance between cfDNA and paired blood samples among the general healthy aging cohort. It was found that $85 \%$ of healthy subjects, $36 \%$ of patients with aplastic anemia and $74 \%$ of those with myelodysplastic syndrome had somatic cfDNA variants, most frequently in DNMT3A, TET2, ASXL1 and SF3B1. Importantly, there was relatively low agreement between clonal fraction of blood as measured by cfDNA and matched blood cells. The cfDNA CH assay used in this study can be applied to detect $\mathrm{CH}$ from historical collections of serial plasma samples. In doing so, this study paves the way for future expanded studies of clonal evolution. However, this study leaves several questions unresolved. In particular, the methodological details of cfDNA isolation and targeted sequencing assays vary considerably from laboratory to laboratory, so it is unclear whether the observations are generally true of other CLIA cfDNA assays. Second, it is unclear whether the clonal fraction estimates from blood and cfDNA are a more reliable estimate of the underlying hematopoietic stem cell clonality that exists in the bone mar- 
row or whether these two estimates have different prognostic relevance.

Taken together, the results from this study show both the promise and the limitations of using cfDNA for studying $\mathrm{CH}$ in research and clinical settings. From a research perspective, the data presented here suggest that it is possible to detect $\mathrm{CH}$ in cfDNA, but rigorous assay validation is required. From a clinical perspective, a physician making a diagnosis of $\mathrm{CH}$ based on a cfDNA test would be wise to perform confirmatory testing in peripheral blood to quantify the clonal fraction accurately, given the discordance between the clonal fraction in cfDNA and blood.

\section{Disclosures}

$A G B$ is a consultant to Foresite Labs.

\section{Contributions}

MAWK and $A G B$ co-wrote the manuscript.

\section{References}

1. Hammond D, Loghavi S. Clonal haematopoiesis of emerging significance. Pathology. 2021;53(3):300-311.

2. Watson CJ, Papula AL, Poon GY, et al. The evolutionary dynamics and fitness landscape of clonal hematopoiesis. Science. 2020;367(6485):1449-1454.

3. Marnell CS, Bick A, Natarajan P. Clonal hematopoiesis of indeterminate potential (CHIP): linking somatic mutations, hematopoiesis, chronic inflammation and cardiovascular disease. J Mol Cell Cardiol. 2021;161:98-105.

4. Nakao T, Bick AG, Taub MA, et al. Bidirectional Mendelian randomization supports bidirectional causality between telomere length and clonal hematopoiesis of intermediate potential. medRxiv. 2021.

https://www.medrxiv.org/content/10.1101/2021.02.26.21252199 [preprint, not peer-reviewed].
5. Nachun D, Lu AT, Bick AG, et al. Clonal hematopoiesis associated with epigenetic aging and clinical outcomes. Aging Cell. 2021;20(6):e13366.

6. Silver AJ, Bick AG, Savona MR. Germline risk of clonal haematopoiesis. Nat Rev Genet. 2021;22(9):603-617.

7. Bhattacharya R, Bick AG. Clonal hematopoiesis of indeterminate potential: an expanding genetic cause of cardiovascular disease. Curr Atheroscler Rep. 2021;23(11):66.

8. Razavi P, Li BT, Brown DN, et al. High-intensity sequencing reveals the sources of plasma circulating cell-free DNA variants. Nat Med. 2019;25(12):1928-1937.

9. Gutierrez-Rodrigues F, Beerman I, Groarke EM, et al. Utility of plasma cell-free DNA for de novo detection and quantification of clonal hematopoiesis. Haematologica. 2022;107(8):1815-1826. 